\title{
Research progress of neurodynamics in China
}

\author{
Rubin Wang ${ }^{1,2} \cdot$ Xiaochuan Pan $^{2}$
}

Published online: 27 February 2021

(C) The Author(s), under exclusive licence to Springer Nature B.V. part of Springer Nature 2021

Since Professor Walter Freeman, a molecular neurobiologist from University of Berkeley, put forward the concept of neurodynamics over 20 years ago (Freeman 2000), the usage of dynamics theories and methods to study cognitive and nervous system activities has become a new field of research (Basar 1998, 2011; Tass 1999; Wang et al. 2015; Wang and Wang 2018; Ravishankar Rao 2018; Haken 1996; Kawato 2000; Takeda 1999; David and Laughlin 2009; Abbott 2008). Research on the topic has sprung up like bamboo shoots. Neurodynamics is more often called computational neuroscience in Europe and the United States, and it is named neuromechanics in Japan (Takeda 1999). Neuroscientists in experimental fields prefer the term neuroinformatics' to describe the basic law of neural information processing qualitatively or quantitatively (Ascoli 2016; Schutter 2016; Raichle and Gusnard 2002). But no matter what name we use, the basic fact remains that neuroscientists and scientists and engineers in the field of artificial intelligence have realized that the development of cognitive neuroscience not only depends on the progress of experimental techniques and data more and more, but also needs to understand and mine underlying principles of dynamical signal processing and transmission in brain networks and the internal mechanism of neural coding distribution mode. The laws and essences behind the vast amount of experimental data, enable us to understand and master the brain's operational methods and the pathogenesis of various brain diseases. An accurate prediction for patients with potential degenerative brain diseases can also be made possible (Laughlin 2001; Chen et al. 2014).

Rubin Wang

rbwang@ecust.edu.cn

1 Key Laboratory of Brain Machine Collaborative Intelligence of Zhejiang Province, Hangzhou Dianzi University, Hangzhou, China

2 Institute for Cognitive Neurodynamics, East China University of Science and Technology, Shanghai, China
Unfortunately, the study in cognitive neuroscience, mainly using experiments as the basic research method, has long focused on finding experimental phenomena, recording experimental data and improving experimental techniques, but pays less attention to the importance of theory. This leads to the fact that brain science, which has a history of hundreds of years, has no systematic and complete theoretical systems of its own. This very abnormal phenomenon makes brain science to date is still an immature subject.

Cognitive neurodynamics is a cross-disciplinary research field that studies cognitive system and nervous system by dynamical theories and methods. Since its inception in 2007, the Journal "Cognitive Neurodynamics" has published a large number of innovative research results by scientists in various research fields from over the world. These published articles have an important impact on the creative construction of brain theory system and brain-like intelligence theory system. One typical example is that we extend the method of modeling dynamic systems in classical mechanics to neuronal systems and creatively construct a new neuron model that is equivalent to the Hodgkin-Huxley (H-H) model (Wang et al. 2015). This new neuron model thus reveals a novel working mechanism to generate neuronal activity that has never been discovered. The novel working mechanism reveals two laws underlying generation of neuronal activity (Wang et al. 2015; Wang and Wang 2018). The first law is that the membrane potential of neurons has a unique corresponding relationship with neuron energy; the second is that neurons mainly consume energy when their membrane potentials are below the threshold, while neurons have both energy absorption and energy consumption when their membrane potentials are above the threshold. The first law indicates the unique correspondence between neural functions of information processing characterized by changing membrane potentials and neural energy (Wang et al. 2015), this finding is strongly confirmed by the $\mathrm{H}-\mathrm{H}$ model (Wang and Wang 2018). The second law could explain an experimental phenomenon that has been puzzled for a long time in neuroscience. The phenomenon is that in fMRI studies, 
the blood flow increases by $31 \%$ after activation of the brain region, while the oxygen consumption increases by only 5\% (Raichle and Gusnard 2002). The new neuron model based on experimental data makes us put forward the concept, theory and method of energy coding (Peng and Wang 2021; Wang et al. 2020). This new concept and coding theory can not only explain some puzzled experimental observations in neuroscience and quantitatively reveal the laws behind some experimental data, but also predict the of phenomena that has not been found in neuroscience (Wang et al. 2020).

Cognitive Neurodynamics published one special issue on "Cognitive Neurodynamics in China" back in 2010. After ten years, we revisit the topic and organized a new special issue of "Research Progress of Neurodynamics in China".

Noticeable progress has been made in the field of Cognitive Neurodynamics in China during these years. Thanks to the diligent work of Chinese scientists, it is a brand new horizon for them to explore and devote researches in this field unceasingly. To promote the communication and cooperation between Chinese scientists and scientists from over the world, seven international conferences on the topic (International Conference on Cognitive Neurodynamics, ICCN) were organized in China and other countries successfully in the past 14 years. In order to deepen the academic exchanges, inspire new ideas and accelerate the cooperation processes, we organize this special issue of Cognitive Neurodynamics. We look forward to your constant concern for these papers, as well as contact with proper authors to discuss the subjects of interest to you.

Through strict peer review, the special issue collected 13 excellent research papers from Chinese scientists together, including one review paper, twelve theoretical and experimental neuroscience papers and one paper on the study of degenerative neurological diseases and cognitive disorders by dynamics theory and methods.

\section{References}

Abbott LF (2008) Theoretical neuroscience rising. Neuron 60(3):489-495

Ascoli GA (2016) On the data-driven road from neurology to neuronomy. Neuroinformatics 14:251-252

Basar E (1998) Brain function and oscillators I: Brain oscillators. Principles and approaches. Springer, Berlin

Basar E (2011) Brain-body-mind in the nebulous cartesian system: a holistic approach by oscillations. Springer, Berlin

Chen M, Guo D, Wang T et al (2014) Bidirectional control of absence seizures by the basal ganglia: a computational evidence. PLoS Comput Biol 10:e1003495

David W, Laughlin MC (2009) Ruling out and ruling in neural codes. Proc Natl Acad Sci PNAS 106(14):5936-5941

De Schutter E (2016) Neuroinformatics for degenerate brains. Neuroinformatics 14:1-3

Freeman WJ (2000) Neurodynamics. Springer, London

Haken H (1996) Principles of brain functioning. Springer, Berlin

Kawato M (2000) The computational theory of brain. Sankyo Press, Tokyo (in Japanese)

Laughlin SB (2001) Energy as a constraint on the coding and processing of sensory information. Curr Opin Neurobiol 11(4):475-480

Peng J, Wang Y, Wang R, Kong W, Zhang J (2021) Neural coupling mechanism in fMRI hemodynamics. Nonlinear Dyn 103:883-895

Raichle ME, Gusnard DA (2002) Appraising the brain's energy budget. Proc Natl Acad Sci PNAS 99(16):10237-10239

Ravishankar Rao A (2018) An oscillatory neural network model that demonstrates the benefits of multisensory learning. Cognit Neurodyn 12(5):481-499

Takeda G (1999) Brain and physics. Shokabo Press, Tokyo (in Japanese)

Tass PA (1999) Phase resetting in medicine and biology. Springer, Berlin

Wang R, Wang Z (2018) The essence of neuronal activity from the consistency of two different neuron models. Nonlinear Dyn 92(3):973-982

Wang R, Tsuda I, Zhang Z (2015) A new work mechanism on neuronal activity. Int J Neural Syst 25(3):1450037

Wang R, Wang Y, Xu X, Pan X (2020) Mechanical thoughts and applications in cognitive neuroscience. Adv Mech 50:450-505 (in Chinese)

Publisher's Note Springer Nature remains neutral with regard to jurisdictional claims in published maps and institutional affiliations. 